\title{
The Cyclization of Alkyl Side Chains of Naphthalenes: The GC/Potential Energies/FTIR Approach
}

\author{
Pavel Straka $^{*}$, Miroslava Novotná ${ }^{2}$, Petr Buryan², Olga Bičáková1 \\ ${ }^{1}$ Institute of Rock Structure and Mechanics, v.v.i., Academy of Sciences of the Czech Republic, Prague, Czech \\ Republic \\ ${ }^{2}$ Institute of Chemical Technology, Prague, Czech Republic \\ Email: ${ }^{*}$ straka@irsm.cas.cz
}

Received 13 August 2014; revised 30 September 2014; accepted 13 October 2014

Copyright (C) 2014 by authors and Scientific Research Publishing Inc.

This work is licensed under the Creative Commons Attribution International License (CC BY).

http://creativecommons.org/licenses/by/4.0/

(c) (i) Open Access

\section{Abstract}

Gas chromatographic measurements of the retention times of alkyl naphthalenes on packed columns with polar and non-polar stationary phases have proven that the logarithm of the relative retention time increases bi-linearly (not linearly) with the number of carbon atoms in a molecule. This is caused by a strong inclination of alkyl side chains toward intramolecular cyclization. A FTIR spectral analysis has shown that longer alkyl side chains of alkyl naphthalenes are cyclized through an interaction between the terminal $\mathrm{CH}_{3}$ group and the aromatic ring. Conventional aromatic-aliphatic molecules thus become new molecules with quasi-alicyclic rings. This, however, alters the effect of non-covalent van der Waals attractive forces both inside and outside the molecules, which is reflected in an exponential increase of the retention times of alkyl naphthalenes with a side chain longer than propyl and in the bi-linearity of the logarithmic dependence of the relative retention times on the number of carbons in the molecule.

\section{Keywords}

Alkyl Naphtalenes, Retention Times, Molecular Mechanics, van der Waals Forces

\section{Introduction}

Classic considerations on the dependence of retention times $\left(R_{t, r e l}\right)$ on the number of carbons in a molecule $(z)$ in different homologous series of gas-chromatographically separated substances always focus on purely linear de-

\footnotetext{
*Corresponding author.
}

How to cite this paper: Straka, P., Novotná, M., Buryan, P. and Bičáková, O. (2014) The Cyclization of Alkyl Side Chains of Naphthalenes: The GC/Potential Energies/FTIR Approach. American Journal of Analytical Chemistry, 5, 957-968. 
pendence without any divergence, be it for isotherm or non-isotherm separations. However, the bi-linearity detected in the dependence $\log R_{t, \text { rel }}=a z+b$ [1] [2] suggests that the behavior of molecules of the compounds in question during separations in stationary phases is more complicated than has been assumed by the classic considerations. The relation $\log R_{t, r e l}=a z+b$ should be expected to be purely linear as a result of the growing number of carbon atoms in the molecule and the corresponding increase of $R_{t, \text { rel }}$ or more precisely $\log R_{t, \text { rel }}$ with the boiling points $\left(T_{b}\right)$ of the given homologs ( $\left.\log R_{t, \text { rel }}=a T_{b}+b\right)$. The mentioned purely linear relation has frequently been applied in the identification of unknown substances using data acquired through gas chromatographic separations, but the validity of this usage is questionable.

In the mentioned studies [1] [2], the relative retention times were expressed as a logarithm of the ratio of the retention time of the given n-alkyl phenol substituted in the ortho, meta and para positions and phenol, and in the case of $n$-alkyl benzenes as a logarithm of the ratio of the retention time of the given n-alkyl benzene and benzene. In the mentioned bi-linearity, a turning point in the increase of the retention characteristics was always observed at $z=9$, i.e. $C_{9}$.

The work [3] investigated cyclization of alkyl side chains. It was deduced that in a high-density stationary phase, the longer alkyl side chains of n-alkyl phenols and n-alkyl benzenes are subject to cyclization as a result of the resistance force of this phase affecting the molecules during their thermal and diffusion motion. Consequently, common conventional aromatic-aliphatic molecules become new molecules with quasi-alicyclic parts. In comparison with the conventionally conceived molecules, the resulting aromatic molecules with quasi-alicyclic parts are likely to be characterized by rather different, possibly even completely different non-covalent interactions between the molecules, chiefly van der Waals interactions, which then affect the retention characteristics. The alkyl side chains of n-alkyl phenols become cyclized in both the polar and nonpolar stationary phases of capillary columns, with a possible formation of hydrogen bridges between the phenolic oxygen and a hydrogen of the methyl group of the alkyl side chain. In the case of n-alkyl benzenes, cyclization is made possible because of the interaction between the benzene ring $(-\delta)$ and a hydrogen of the terminal methyl group $(+\delta)$ of the alkyl chain (Figure 1(a) and Figure 1(b)).

In the case of the formed aromatic molecules with quasi-alicyclic rings, the effect of van der Waals forces thus increases not only intramolecularly but also intermolecularly. In the case of n-alkyl phenols and n-alkyl benzenes, this effect is strong with the molecules having the number of carbons higher than nine. This results in bi-linearity in the retention characteristics of these compounds, observed in the dependence of the logarithm of the relative retention time on the total number of carbons in the molecule. Yet it is not clear whether the cyclization of the side chains of alkyl phenols and alkyl benzenes is their inherent feature or it occurs only during their motion through the column.

The mentioned phenomena can be evaluated and compared using methods of computational chemistry. In order to assess cyclization, association and different interactions, one can compute the energies of the covalent bonds and non-covalent, mainly van der Waals, interactions. For the purpose of this assessment, one can also estimate molecular conformations which are realistic and energetically advantageous or possible and are in correlation with the ascertained chromatographic data. In our case, on the basis of experience with the evaluation of aromatic, aromatic-aliphatic, aromatic-alicyclic and phenolic structures in terms of energy using the methods of molecular mechanics [3], two methods of molecular mechanics were selected for the calculations: the force fields $\mathrm{MM}+$ and AMBER.

In this work, the question of the cyclization of the alkyl side chains as an inherent property of aromatic-aliphatic molecules is resolved on the basis of the FTIR spectra interpretation of n-alkyl phenols as model compounds.

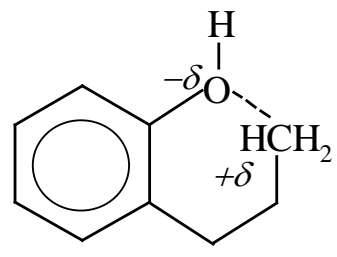

(a)

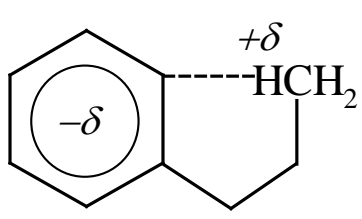

(b)

Figure 1. Concepts of the quasi-cyclized forms in the case of ortho n-propyl phenol (a) and n-propyl benzene (b). The cyclization is realized on the terminal methyl group. 
Another aim of the presented work is to explain the bi-linearity in the logarithmic dependence of the relative retention times on the number of carbons in the molecule in the case of n-alkyl naphthalenes by molecular mechanics modeling and to clarify the behavior of n-alkyl naphthalenes in the environment of different stationary phases of packed GC columns. For the calculations, the potential energies of the bonds and non-covalent interactions have been considered.

\section{Experimental}

\subsection{Chemicals}

For the FTIR spectral study, 2-n-ethyl phenol and 2-n-butyl phenol (analytical grade, Sigma Aldrich) were used. For the GC measurements of n-alkyl naphthalenes, a) 1-methyl-, 1-ethyl-, 1-n-propyl-, 1-n-butyl- and 1-n-hexylnaphthalenes, b) 2-methyl-, 2-ethyl-, 2-n-propyl-, 2-n-butyl- and 2-n-pentyl-naphthalenes (GC grade, Lach-Ner SRO, Czech Rep.) were utilized. Where necessary, naphthalenes were diluted with isooctane.

\subsection{Gas Chromatography}

Gas chromatographic measurements were carried out on packed columns (1.2 m, an internal diameter of $2 \mathrm{~mm}$ ) with stationary phases of 2.5\% liquid crystals BMBT on 100/120 Chrom W-HP (Alltech Associates) (polar phase [4] [5]) and 3\% OV-17 on Chromaton N 0.125 - 0.160 mm (Erba Lachema SRO) (non-polar phase [6]). On the polar phase BMBT, the measurements of the retention times of both 1-n-alkyl naphthalenes $\left(C_{11}-C_{16}\right)$ and 2-n-alkyl naphthalenes $\left(C_{11}-C_{15}\right)$ were performed at a temperature of $80^{\circ} \mathrm{C}$; on the nonpolar phase $\mathrm{OV}-17$, these alkyl naphthalenes were measured at a temperature of $110^{\circ} \mathrm{C}$. The other conditions of the experiments were as follows: liquid injector: $200^{\circ} \mathrm{C}$; carrier gas: nitrogen, $40 \mathrm{ml} / \mathrm{min}$; FID: hydrogen, $30 \mathrm{ml} / \mathrm{min}$, air, 400 $\mathrm{ml} / \mathrm{min}$. The relative retention times $\left(R_{t, r e l}\right)$ were expressed as logarithms of the ratio of the retention time of the given n-alkyl naphthalene and naphthalene, $\left(R_{t, \text { n-alkylnaphthalene }} / R_{t, \text { naphthalene }}\right)$. GC measurements were carried out on a gas chromatograph Chrom 5 (Laboratorní přistroje Praha, Czech Rep.).

\subsection{FTIR Spectroscopy}

Samples of 2-ethyl phenol and 2-n-butyl phenol were measured using the capillary thin film method while liquid samples were placed between two $\mathrm{NaCl}$ windows. FTIR spectra were acquired on a FTIR spectrometer Nicolet 250 (Nicolet Instruments Co., USA). A KBr beam splitter and a DTGS detector were used. 132 scans were collected for each measurement over the spectral range of $400-4000 \mathrm{~cm}^{-1}$ with a resolution of $4 \mathrm{~cm}^{-1}$; for apodization, a Happ-Genzel function was used. The spectra were processed using Omnic 6.1 software (Nicolet Instruments Co., USA).

\subsection{Calculations}

Calculations were done with molecular mechanics methods [7]-[11] using force fields; based on the mentioned previous experience with n-alkyl benzenes, MM+ and AMBER methods were utilized. The reason is that these methods use analytical and relatively simple potential energy functions for describing the interactions between a set of atoms; further, they are empirical, accurate and very suitable for small organic molecules. It is important that the fundamental basis for calculating, the interactions are not atoms but atom types. In these methods, the interaction potential describes both bonding and non-bonding interactions. In the potential, the following energetic terms were calculated:

- bond stretching ( $E_{\mathrm{bond}}$ ), which is associated with the deformation of a bond from its standard equilibrium length;

- bond angle bending ( $E_{\text {angle }}$, which is associated with the deformation of an angle from its normal value;

- stretch-bend ( $E_{\text {stretch-bend }}$ — the bond-stretching and angle-bending cross term, which includes coupling between the bond stretching and angle bending;

- dihedrals $\left(E_{\text {dihedral }}\right)$ - torsional energy, which is associated with the tendency of dihedral angles to have a certain $n$-fold symmetry and to have minimum energy;

- van der Waals ( $\left.E_{\text {vdWaals }}\right)$, which describes the repulsive forces keeping two non-bonded atoms apart at close range and the attractive forces drawing them together at long range; 
- electrostatic $\left(E_{\text {elst }}\right)$, which describes non-bonded electrostatic interactions, particularly dipole-dipole interations.

These energetic terms were calculated using both the MM+ and AMBER methods, with the exception of

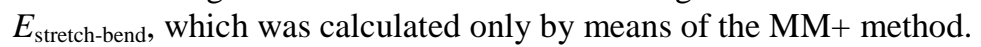

The mentioned potential energies of the covalent bonds and non-covalent interactions were calculated for common (conventional) n-alkyl naphthalenes as well as for the models of the cyclized forms of these compounds. The concepts of the cyclized forms were formulated on the basis of the study of the distribution of electron densities (atomic charges in a molecule, [8]) in common and cyclized molecules. In the case of the cyclized form of n-alkyl naphthalenes, due to a higher electronegativity of carbon in comparison with that of hydrogen (2.6 and 2.2, respectively, the Pauling scale), the electron density on the methyl carbon was discovered to be considerably higher than on the other alkyl carbons. Consequently, the hydrogen atom bonded with this carbon has a $+\delta$ charge. The closing of the alicyclic ring was thus implemented by an interaction of a methyl hydrogen with the ascribed charge $+\delta$ and the naphthalene ring with the ascribed charge $-\delta$. The concepts of cyclized forms and the models of these forms for the sake of calculations are shown in Figures 2(a)-(d) for 1-n-propyl naphthalene and 1-n-butyl naphthalene. The cyclized forms and models for other 1-n-alkyl naphthalenes and 2-alkyl naphthalenes were designed similarly. The n-alkyl naphthalenes considered were cyclized to the ortho position with respect to the alkyl. Generally, cyclization is always implemented on the terminal methyl group.

\section{Results and Discussion}

\subsection{GC Measurements Results}

The following considerations presented are based on gas chromatographic measurements on packed columns with both polar and nonpolar stationary phases. The measurements of the relative retention times $\left(R_{t, \text { rel }}\right)$ of n-alkyl naphthalenes $C_{11}-C_{16}$ on these columns showed that the logarithms of these retention times increase bi-linearly with the number of carbon atoms in a molecule (z) (Figures 3-6). Two linear areas in the consecutive intervals $C_{11}-C_{13}$ and $C_{13}-C_{16}$, with the slopes of each line being different, were proven in the relation $\log R_{t, \text { rel }}$ $=a z+b$ (where $a$ and $b$ are constants), although only one line had been anticipated to be found. The complete dependence was thus of a bi-linear character. The turning point in the bi-linearity was always observed when the propyl was a substituent. The same phenomenon was observed with n-alkyl benzenes and n-alkyl phenols [3].

\subsection{The Potential Energies of Bonds and Non-Covalent Interactions}

It arises from Figures 3-6 that the chromatographic behavior of the alkyl naphthalenes studied is independent of the polarity of the stationary phase, because the polar and nonpolar phases provided the same bi-linear dependence. This implies that the significant increase in the retention times of alkyl naphthalenes with a chain longer than propyl is rather connected with the physical properties of the molecules than with their chemical structure. Therefore, the potential energies of the bonds and of non-covalent, namely van der Waals, interactions were computed and compared. It was also taken into account that the considered cyclization leads to a decrease in molecular size, which may facilitate the mixing of alkyl naphthalene molecules with the stationary phase by reducing the enthalpy of mixing $\left(\Delta \mathrm{H}_{\text {mix }}\right)$, in an ideal case even to the thermodynamic condition $\Delta \mathrm{H}_{\text {mix }}=0$ (Consequently, $\Delta \mathrm{G}_{\text {mix }}=\Delta \mathrm{H}_{\text {mix }}-\mathrm{T} \Delta \mathrm{S}_{\text {mix }}<0$, when the mixing and hence also the solubility of alkyl naphthalenes in the stationary phase reach their maximum). The longer a side chain is, the more significantly its cyclization contributes to mixing. The stationary phase-cyclized alkyl naphthalene system is thermodynamically more stable, which may be a reason for the preference of cyclized structures over conventional ones.

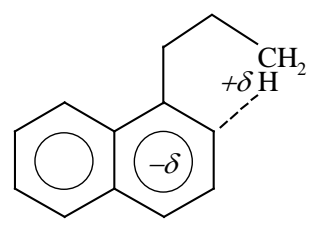

(a)

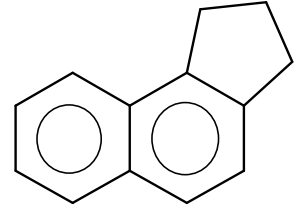

(b)

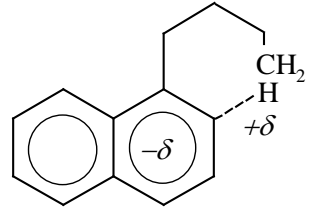

(c)

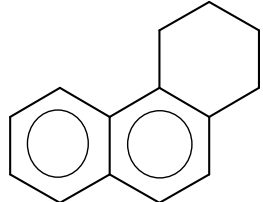

(d)

Figure 2. Concepts of the quasi-cyclized forms in the case of 1-n-propyl naphthalene (a) and 1-n-butyl naphthalene (c). Cyclization is realized on the terminal methyl group. The concepts (b) and (d) are the models for energy calculations; (b) The model of cyclized 1-n-propyl naphthalene; (d) The model of cyclized 1-n-butyl naphthalene. 


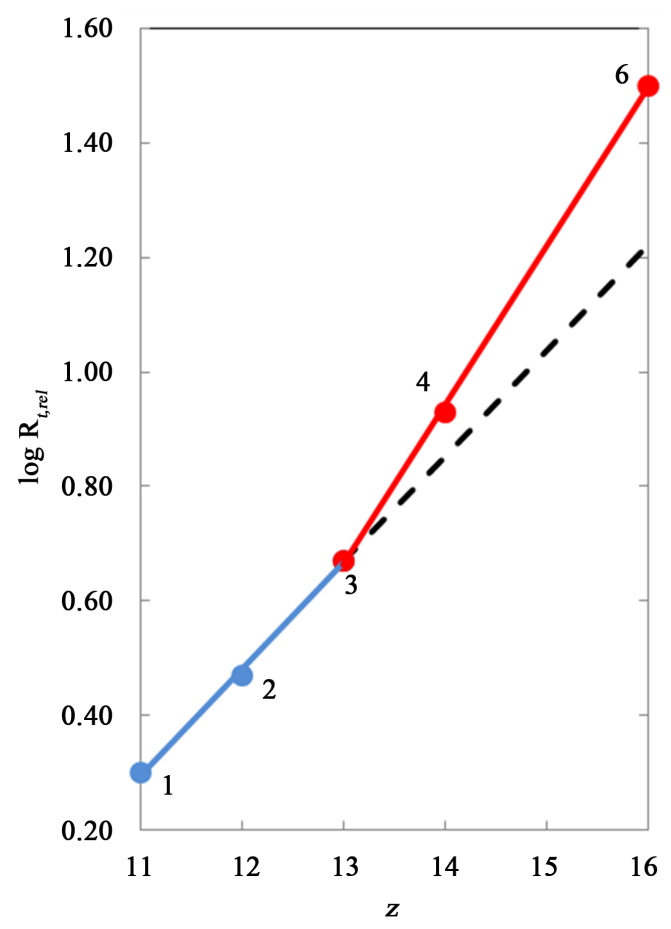

Figure 3. The bi-linear dependence of $\log R_{t, \text { rel }}$ on the number of carbons in the molecule (z) for 1-n-alkyl naphtalenes. ( $R_{t, \text { rel }}$ the relative retention time.) 1,2 , 3 1-methyl-, 1-ethyl-, 1-n-propyl naphtalenes, resp. (blue line), 4, 6-1-n-butyl-, 1-nhexyl naphtalenes, resp. (red line) (The polar phase BMBT, $80^{\circ} \mathrm{C}$ ).

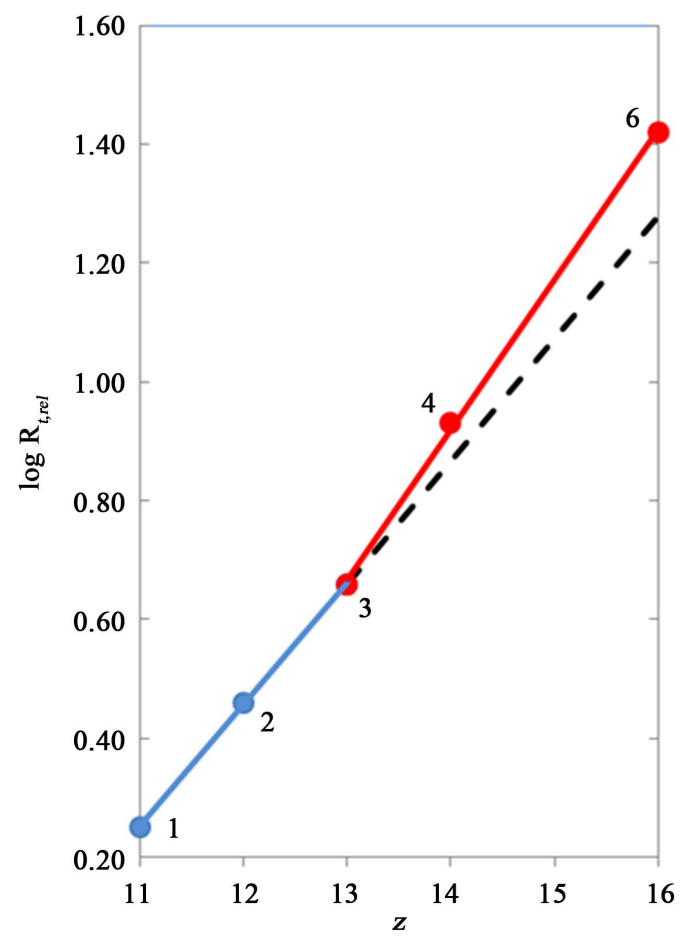

Figure 4. The bi-linear dependence of $\log R_{t, r e l}$ on the number of carbons in the molecule (z) for 1-n-alkyl naphtalenes. $\left(R_{t, \text { rel }}\right.$ the relative retention time.) 1,2 , 3 1-methyl-, 1-ethyl-, 1-n-propyl naphtalenes, resp. (blue line), 4, 6-1-n-butyl-, 1-nhexyl naphtalenes, resp. (red line) (The nonpolar phase $\mathrm{OV}-17,110^{\circ} \mathrm{C}$ ). 


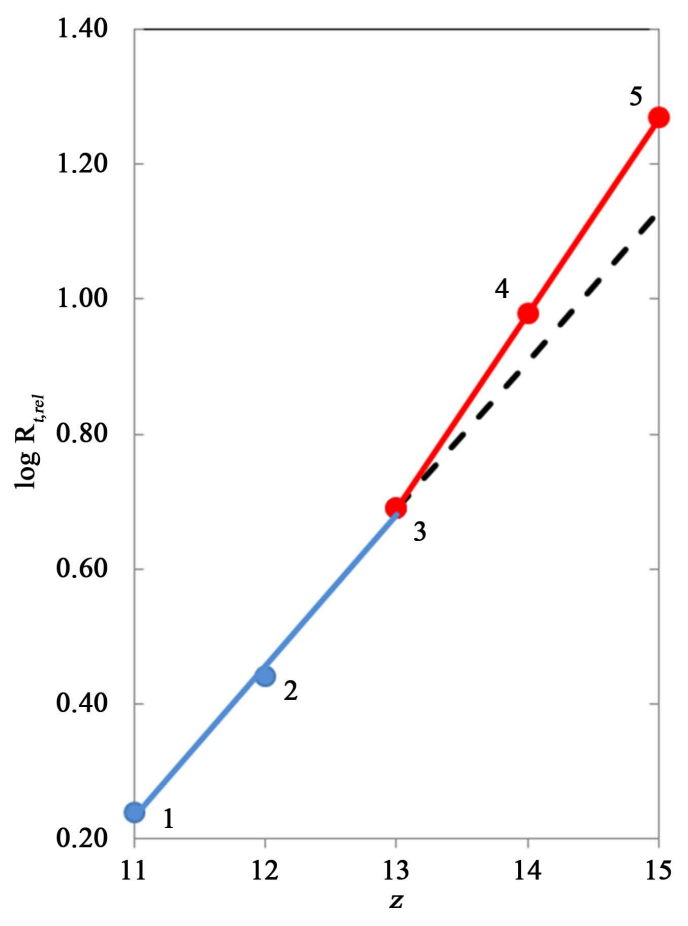

Figure 5. The bi-linear dependence of $\log R_{t, \text { rel }}$ on the number of carbons in the molecule $(z)$ for 2 -n-alkyl naphtalenes. $\left(R_{t, \text { rel }}\right.$-the relative retention time.) $1,2,3$ 2-methyl-, 2-ethyl-, 2-n-propyl naphtalenes, resp. (blue line), 4, 5-2-n-butyl-, 2-npentyl naphtalenes, resp. (red line) (The polar phase BMBT, $80^{\circ} \mathrm{C}$ ).

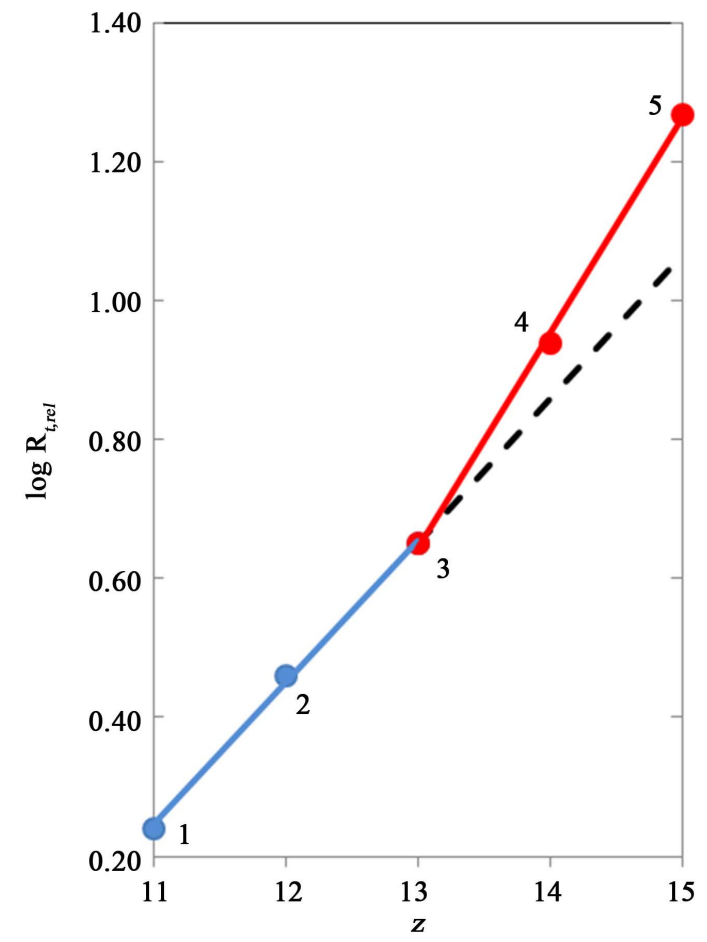

Figure 6. The bi-linear dependence of $\log R_{t, \text { rel }}$ on the number of carbons in the molecule $(z)$ for 2-n-alkyl naphtalenes. $\left(R_{t, r e l}\right.$ the relative retention time.) 1,2 , 32-methyl-, 2-ethyl-, 2-n-propyl naphtalenes, resp. (blue line), 4, 5-2-n-butyl-, 2-n-pentyl naphtalenes, resp. (red line) (The nonpolar phase $\mathrm{OV}-17,110^{\circ} \mathrm{C}$ ). 
The computed potential energies of covalent bonds and van der Waals interactions between two non-bonded atoms in molecules of 1-n-alkyl naphthalenes and 2-n-alkyl naphthalenes have been summarized in Tables 1-4. The energies of electrostatic, specifically dipole-dipole, interactions were always zero as expected. The energies of the bonds and interactions were calculated for conventional molecules and molecules cyclized into the ortho position with respect to the alkyl substituent. The cyclization was considered to have occurred on the terminal methyl group.

With conventional molecules of the alkyl naphthalenes studied, neither an increase in the number of carbons nor the growing length of the alkyl chain led to a significant change in potential energies. Minor changes were recorded in $E_{\text {bond }}$ and $E_{\text {vdWaals }}$ values; all the other values remained constant or almost constant. With cyclized molecules, on the other hand, the values of all potential energies and interactions with the exception of $E_{\text {elst }}$

Table 1. Potential energies of covalent bonds and non-covalent interactions in 1-n-alkyl naphthalenes, common and cyclized into the position ortho with respect to the alkyl substituent (cyclization on $\mathrm{CH}_{3}$ ). Calculated by the $\mathrm{MM}+$ program.

\begin{tabular}{ccccccc}
\hline & $\boldsymbol{E}_{\text {bond }}$ & $\boldsymbol{E}_{\text {angle }}$ & $\boldsymbol{E}_{\text {stretch-bend }}$ & $\boldsymbol{E}_{\text {dihedral }}$ & $\boldsymbol{E}_{\text {vdWaals }}$ & $\boldsymbol{E}_{\text {elst }}$ \\
\cline { 2 - 7 } 1-n-alkyl naphtalenes & & & & & \\
Naphtalene & 6.78 & 0.00 & 0.00 & -64.48 & 26.63 & \\
Common: & & & & & \\
Methyl naphtalene & 9.13 & 0.25 & -0.13 & -64.19 & 36.09 & 0 \\
Ethyl naphtalene & 11.01 & 0.25 & -0.13 & -64.77 & 60.75 & 0 \\
n-Propyl naphtalene & 12.90 & 0.25 & -0.13 & -64.77 & 56.06 & 0 \\
n-Butyl naphtalene & 14.82 & 0.25 & -0.13 & -64.77 & 66.57 & 0 \\
n-Pentyl naphtalene & 16.71 & 0.25 & -0.13 & -64.77 & 69.76 & 0 \\
n-Hexyl naphthalene & 18.63 & 0.25 & -0.08 & -64.77 & 72.94 & 0 \\
Cyclized on CH 3 : & & & & & \\
Methyl naphtalene & 9.71 & 949.23 & -8.00 & 126.03 & 23.03 & 0 \\
Ethyl naphtalene & 30.57 & 137.67 & -0.42 & -51.58 & 22.07 & 0 \\
Propyl naphtalene & 14.61 & 16.20 & -0.33 & -48.11 & 28.60 & 0 \\
Butyl naphtalene & 14.24 & 1.30 & -0.17 & -62.47 & 56.86 & 0 \\
Pentyl naphtalene & 16.37 & 2.22 & -0.08 & -47.15 & 117.03 & 0 \\
Hexyl naphthalene & 18.05 & 0.75 & -0.21 & -34.79 & 514.46 & 0 \\
\hline
\end{tabular}

Table 2. Potential energies of covalent bonds and non-covalent interactions in 1-n-alkyl naphtalenes, common and cyclized into the position ortho with respect to the substituent (cyclization on $\mathrm{CH}_{3}$ ). Calculated by the AMBER program.

\begin{tabular}{|c|c|c|c|c|c|}
\hline \multirow{2}{*}{ 1-n-alkyl naphtalenes } & $E_{\text {bond }}$ & $\boldsymbol{E}_{\text {angle }}$ & $\boldsymbol{E}_{\text {dihedral }}$ & $\boldsymbol{E}_{\mathrm{vdWaals}}$ & $E_{\text {elst }}$ \\
\hline & \multicolumn{5}{|c|}{$(\mathrm{kJ} / \mathrm{mol})$} \\
\hline Naphtalene & 0.00 & 0.00 & 0.00 & 24.54 & 0 \\
\hline \multicolumn{6}{|l|}{ Common: } \\
\hline Methyl naphtalene & 0.13 & 0.00 & 0.00 & 31.74 & 0 \\
\hline Ethyl naphtalene & 0.38 & 1.63 & 0.00 & 52.97 & 0 \\
\hline n-Propyl naphtalene & 0.63 & 1.63 & 0.00 & 52.04 & 0 \\
\hline n-Butyl naphtalene & 0.88 & 1.63 & 0.00 & 53.84 & 0 \\
\hline n-Pentyl naphtalene & 1.13 & 1.63 & 0.00 & 54.60 & 0 \\
\hline n-Hexyl naphthalene & 1.42 & 1.63 & 0.00 & 55.39 & 0 \\
\hline \multicolumn{6}{|l|}{ Cyclized on $\mathrm{CH}_{3}$ : } \\
\hline Methyl naphtalene & 1.42 & 626.21 & 58.41 & 23.24 & 0 \\
\hline Ethyl naphtalene & 2.18 & 348.90 & 10.68 & 23.41 & 0 \\
\hline Propyl naphtalene & 3.85 & 40.74 & 19.18 & 23.24 & 0 \\
\hline Butyl naphtalene & 1.09 & 2.68 & 1.80 & 46.22 & 0 \\
\hline Pentyl naphtalene & 1.30 & 4.23 & 14.99 & 156.09 & 0 \\
\hline Hexyl naphthalene & 1.72 & 4.65 & 28.30 & 6188.39 & 0 \\
\hline
\end{tabular}


Table 3. Potential energies of covalent bonds and non-covalent interactions in 2-n-alkyl naphtalenes, common and cyclized into the position ortho with respect to the substituent (cyclization on $\mathrm{CH}_{3}$ ). Calculated by the $\mathrm{MM}+$ program.

\begin{tabular}{ccccccc}
\hline & $\boldsymbol{E}_{\text {bond }}$ & $\boldsymbol{E}_{\text {angle }}$ & $\boldsymbol{E}_{\text {stretch-bend }}$ & $\boldsymbol{E}_{\text {dihedral }}$ & $\boldsymbol{E}_{\text {vdWaals }}$ & $\boldsymbol{E}_{\text {elst }}$ \\
\cline { 2 - 7 } 2-n-alkyl naphtalenes & & & & & \\
Naphtalene & 6.78 & 0.00 & 0.00 & 24.64 & 26.63 & \\
Common: & & & & & \\
Methyl naphtalene & 9.13 & 0.25 & -0.13 & -67.49 & 28.93 & 0 \\
Ethyl naphtalene & 11.01 & 0.25 & -0.13 & -68.08 & 53.80 & 0 \\
n-Propyl naphtalene & 12.90 & 0.25 & -0.13 & -68.08 & 56.82 & 0 \\
n-Butyl naphtalene & 14.82 & 0.25 & -0.13 & -68.08 & 59.79 & 0 \\
n-Pentyl naphtalene & 16.71 & 0.25 & -0.13 & -68.08 & 62.97 & 0 \\
n-Hexyl naphthalene & 18.63 & 0.25 & -0.13 & -68.08 & 66.15 & 0 \\
Cyclized on CH ${ }_{3}:$ & & & & & \\
Methyl naphtalene & 8.67 & 955.35 & -7.49 & 121.59 & 24.03 & 0 \\
Ethyl naphtalene & 30.98 & 137.17 & -0.59 & -54.89 & 24.62 & 0 \\
Propyl naphtalene & 14.65 & 16.20 & -0.33 & -51.42 & 28.01 & 0 \\
Butyl naphtalene & 14.28 & 1.26 & -0.17 & -65.74 & 41.58 & 0 \\
Pentyl naphtalene & 16.12 & 2.01 & -0.04 & -51.33 & 78.00 & 0 \\
Hexyl naphthalene & 18.00 & 1.42 & -0.04 & -48.49 & 96.59 & 0 \\
\hline
\end{tabular}

Table 4. Potential energies of covalent bonds and non-covalent interactions in 2-n-alkyl naphtalenes, common and cyclized into the position ortho with respect to the substituent (cyclization on $\mathrm{CH}_{3}$ ). Calculated by the AMBER program.

\begin{tabular}{|c|c|c|c|c|c|}
\hline \multirow{2}{*}{ 2-n-alkyl naphtalenes } & $E_{\text {bond }}$ & $E_{\text {angle }}$ & $E_{\text {dihedral }}$ & $E_{\text {vdWaals }}$ & $E_{\text {elst }}$ \\
\hline & \multicolumn{5}{|c|}{$(\mathrm{kJ} / \mathrm{mol})$} \\
\hline Naphtalene & 0.00 & 0.00 & 0.00 & 24.54 & 0 \\
\hline \multicolumn{6}{|l|}{ Common: } \\
\hline Methyl naphtalene & 0.13 & 0.00 & 0.00 & 25.71 & 0 \\
\hline Ethyl naphtalene & 0.38 & 1.63 & 0.00 & 47.19 & 0 \\
\hline n-Propyl naphtalene & 0.63 & 1.63 & 0.00 & 47.61 & 0 \\
\hline n-Butyl naphtalene & 0.88 & 1.63 & 0.00 & 48.19 & 0 \\
\hline n-Pentyl naphtalene & 1.13 & 1.63 & 0.00 & 48.95 & 0 \\
\hline n-Hexyl naphthalene & 1.42 & 1.63 & 0.00 & 49.78 & 0 \\
\hline \multicolumn{6}{|l|}{ Cyclized on $\mathrm{CH}_{3}$ : } \\
\hline Methyl naphtalene & - & 620.35 & 57.53 & 24.12 & 0 \\
\hline Ethyl naphtalene & 19.18 & 347.86 & 10.68 & 24.79 & 0 \\
\hline Propyl naphtalene & 3.85 & 40.61 & 19.13 & 22.69 & 0 \\
\hline Butyl naphtalene & 1.00 & 2.68 & 1.80 & 28.47 & 0 \\
\hline Pentyl naphtalene & 1.84 & 5.78 & 12.31 & 72.31 & 0 \\
\hline Hexyl naphthalene & 1.72 & 6.24 & 14.57 & 107.52 & 0 \\
\hline
\end{tabular}

changed. Considerably different $E_{\text {angle }}$ values were exhibited by cyclized methyl- and ethyl naphthalenes, which is connected with a significant strain in three- and four-membered hypothetical quasi-alicyclic rings. Since such cyclization reactions do not occur in reality, these values do not have to be taken into account. In the cases of 1and 2-methyl naphthalenes, the $E_{\text {dihedral }}$ values calculated using the MM+ method were also substantially different (Table 1 and Table 3, the column $E_{\text {dihedral }}$ ). For the above-mentioned reason, however, not even these values need to be taken into consideration. In the other cases, there was no dependence of the potential energy on the number of carbons in a molecule and it may be said that these are uncorrelated quantities. In contrast, there was a strong dependence of the energy of van der Waals interactions on the number of carbons in a molecule, both in 1-n- and in 2-n-alkyl naphthalenes. For $z$ being 11 - 13, these forces change only little or not at all, but when $z$ is in the range of 13 - 16, the acting intramolecular van der Waals forces significantly increase. This is illustrated 
well in Figure 7. As a result, the dependences obtained had a bi-linear character, like the mentioned dependences of $\log R_{t, \text { rel }}$ on $z$; the turning point occurred at $z>13$ (Tables 1-4, columns $E_{\text {vdWaals }}$ ), that is at the propyl substituent. It may thus be inferred that the bi-linear character of this dependence is causally related to intramolecular van der Waals forces.

The increase of van der Waals forces acting inside a molecule must necessarily be reflected also intermolecularly. Between the cyclized molecules, there are hence attractive forces, which increase with the length of an alkyl chain and whose effect in the case of quasi-alicyclic rings from butyl, pentyl and hexyl substituents is strong. This leads to an exponential increase in retention times with the length of the alkyl chain and to the bi-linearity in the logarithmic dependence of the relative retention times with a steeper connecting line of $\log R_{t, \text { rel }}$ for butyl, pentyl and hexyl substituents.

\subsection{The Results of FTIR Measurements}

A key issue in the study of the cyclization of side chains is whether it occurs during their motion through the dense medium of the chromatographic column or it is an inherent feature of alkyl chains attached to an aromatic structure.

The possibility of the cyclization of alkyl side chains as an inherent feature of the chains was studied with n-alkyl phenols, whose infrared spectra should reflect well the interactions of these chains with the aromatic ring. As mentioned, the interaction should take place on the terminal $\mathrm{CH}_{3}$ group [3]. The FTIR spectra of 2-ethyl phenol and 2-n-butyl phenol were compared. In the case of 2-ethyl phenol, cyclization was not expected because of the high strain in the formed quasi-alicyclic ring; in 2-n-butyl phenol, on the other hand, cyclization was considered to be quite realistic because of the formation of a six-membered quasi-alicyclic ring, which is very stable. The infrared spectra of the thin films of liquid samples clearly proved differences in the spectral region of 3150 - 2980 $\mathrm{cm}^{-1}$, i.e. in the region of the symmetric and asymmetric stretching vibrations of $\mathrm{C}-\mathrm{H}$ in $\mathrm{CH}_{3}$ and $\mathrm{CH}_{2}$ groups attached to an aromatic ring (Figure 8). A significant change is the absorption band at $2996 \mathrm{~cm}^{-1}$, observed for 2-n-butyl but absent with 2-ethyl phenol. This band may be ascribed to an interaction of the methyl group of the butyl chain with an aromatic ring, leading to the formation of a quasi-alicyclic ring (Figure 2(c)), more precisely an interaction of the hydrogen atom of the terminal methyl group with a $+\delta$ charge with the aromatic ring with a $-\delta$ charge. The differences in other absorption bands, in the spectral region of $2980-2750 \mathrm{~cm}^{-1}$, are caused by the different lengths of the alkyl chains of the alkyl phenols observed.

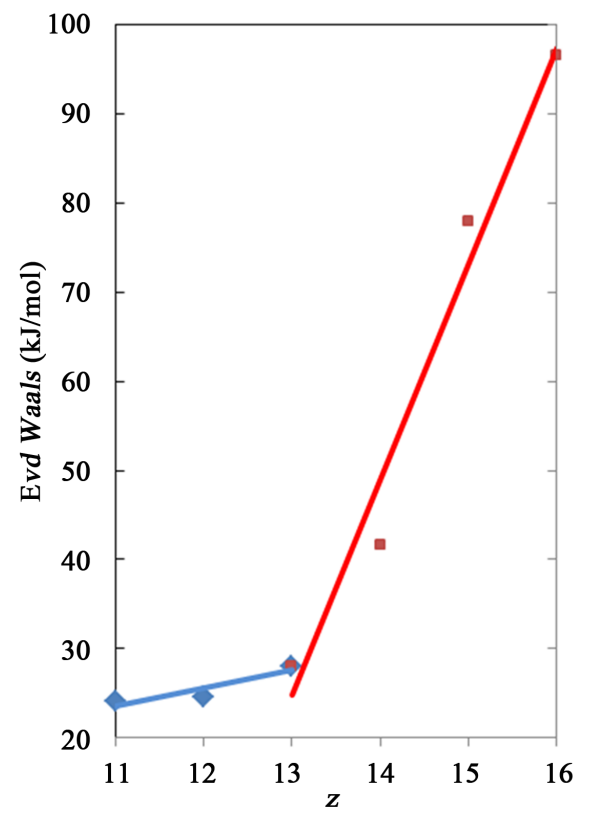

Figure 7. The dependence of the van der Waals energies (and consequently forces) on the number of carbons in the cyclized molecules of 2-n-alkyl naphthalenes (the MM+ method). 


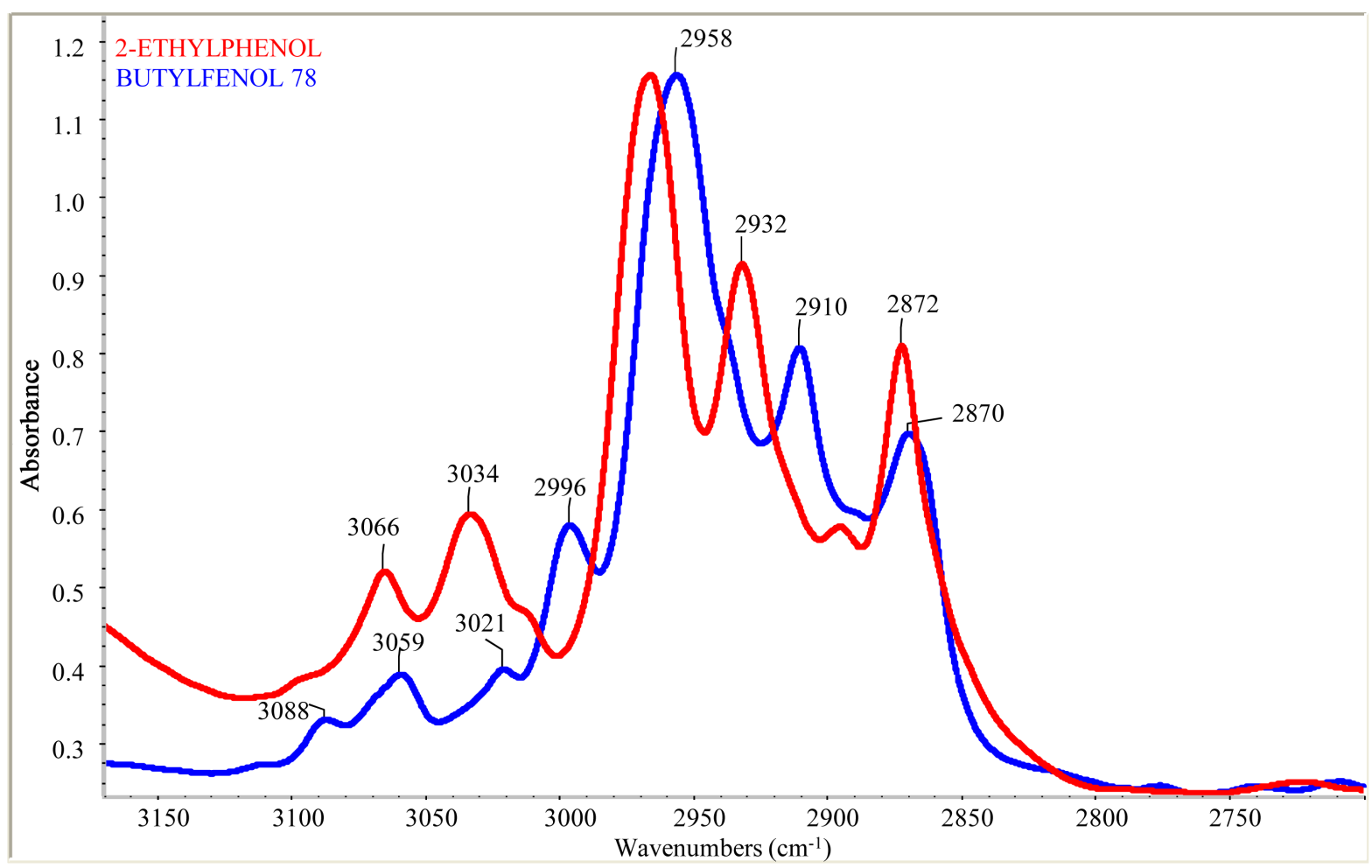

Figure 8. The FTIR spectra of 2-ethyl phenol (red line) and 2-n-butyl phenol (blue line) in the spectral region of 3150 - 2750 $\mathrm{cm}^{-1}$ with a key interval of $3100-2980 \mathrm{~cm}^{-1}$ (a thin film between $\mathrm{NaCl}$ windows).

The methyl-group interaction being considered is also supported by a shift of the absorption band of the stretching vibration of $\mathrm{C}=\mathrm{C}$ in the aromatic ring observed in the region of $1680-1530 \mathrm{~cm}^{-1}$ (Figure 9). The band recorded in the case of 2-ethyl phenol at $1591 \mathrm{~cm}^{-1}$ was shifted to $1582 \mathrm{~cm}^{-1}$ for 2-n-butyl phenol and complemented by another band at $1651 \mathrm{~cm}^{-1}$, which had not been observed for 2-ethyl phenol. These differences are attributable to changes in the electron density of the aromatic ring as a consequence of the attachment of a butyl to this ring and the formation of a quasi-cyclic structure (Figure 10).

Another difference was observed in the region of the deformation vibrations of $\mathrm{C}=\mathrm{C}-\mathrm{C}$ in the plane of the aromatic ring of $680-505 \mathrm{~cm}^{-1}$. For 2-n-butyl phenol, a band was discovered here at $543 \mathrm{~cm}^{-1}$ (Figure 11); it does not occur in the case of 2-ethyl phenol and it also can be ascribed to the considered interaction of the $\mathrm{CH}_{3}$ group of the butyl chain with the aromatic ring.

In addition, dilution experiments were performed with the aim to prove that the interaction in question is intramolecular. The relative intensity of the absorption bands in the spectral region of $3150-2900 \mathrm{~cm}^{-1}$ (i.e. in the region of the symmetric and asymmetric stretching vibrations of $\mathrm{C}-\mathrm{H}$ in $\mathrm{CH}_{3}$ and $\mathrm{CH}_{2}$ groups attached to an aromatic ring) was monitored in dependence on the dilution of alkyl phenol by carbon disulfide. The spectra of the solutions were studied in measuring cells of 0.1 and $1 \mathrm{~mm}$. In the case of neither 2-ethyl phenol nor 2-n-butyl phenol did the spectra obtained show any significant changes after the samples were diluted. It can thus be clearly concluded that the interaction of a butyl chain with an aromatic ring is intramolecular; otherwise, the relative intensity of the studied absorption bands would have changed with increasing dilution. The cyclization of alkyl side chains by means of an intramolecular reaction of the terminal methyl group with an aromatic ring hence seems to be an inherent feature of alkyl-substituted aromatic compounds.

In summary, intramolecular cyclization is accompanied by a decrease in the effective size of molecules, which is significant for $C_{13}$ and larger molecules. Aromatic quasi-alicyclic molecules of a reduced size are more easily mixed with the dense stationary phase, and the formed system is, in comparison with a system with common aromatic-aliphatic molecules, more homogenous and thus thermodynamically more stable. The change in the size of the molecules is then accompanied by changes in van der Waals interactions and subsequently by a change in the relative retention time, because an increase in the van der Waals interactions inside the cyclized molecules 


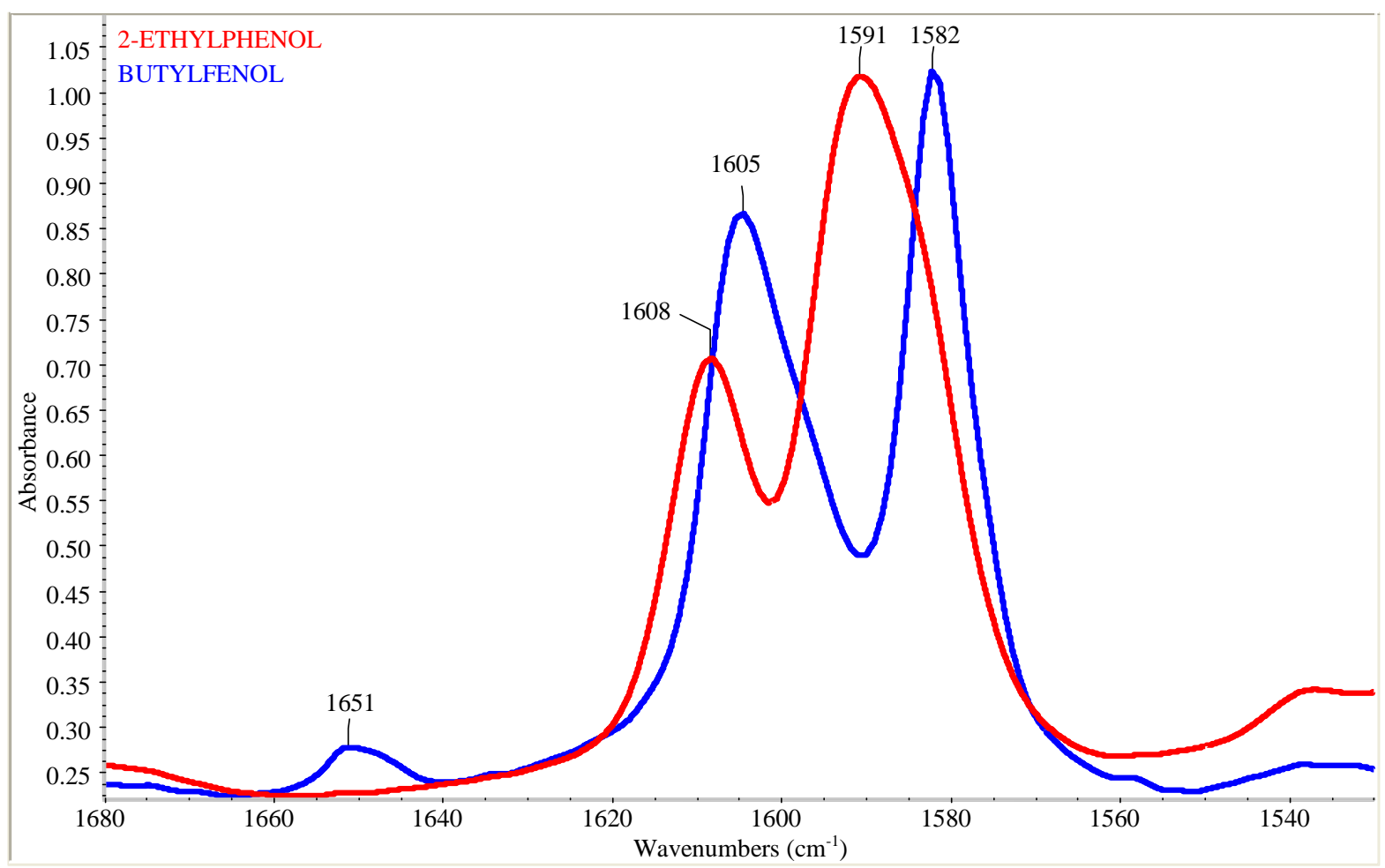

Figure 9. The FTIR spectra of 2-ethyl phenol (red line) and 2-n-butyl phenol (blue line) in the spectral region of 1680 - 1530 $\mathrm{cm}^{-1}$ (a thin film between $\mathrm{NaCl}$ windows).<smiles>Oc1cccc2c1CCCC2</smiles>

Figure 10. The quasi-alicyclic structure of 2-nbutyl phenol.

must also be reflected in an increase of these forces between the molecules as a more intense effect of the attraction forces between the molecules.

\section{Conclusion}

The alkyl side chains of n-alkyl naphthalenes become cyclized, as demonstrated using FTIR spectroscopy. Cyclization is made possible because of the intramolecular interaction between the aromatic ring $(-\delta$ and a hydrogen of the terminal methyl group $(+\delta$ of the alkyl chain. With the formed aromatic molecules with a quasi-alicyclic ring, the effect of the van der Waals forces increases not only intramolecularly but also intermolecularly. In the case of n-alkyl naphthalenes, this effect is strong with molecules having the number of carbons in the molecule higher than thirteen. This results in bi-linearity in the retention characteristics of these compounds, observed in the dependence of the logarithm of the relative retention time on the total number of carbons in the molecule in both the polar and nonpolar stationary phases of packed columns. The role of van der Waals forces was demonstrated using the potentials energies of the covalent bonds and non-covalent interactions for 1-n-alkyl naphthalenes and 2-n-alkyl naphthalenes, both common and cyclized into the ortho position with respect to the substituent. 


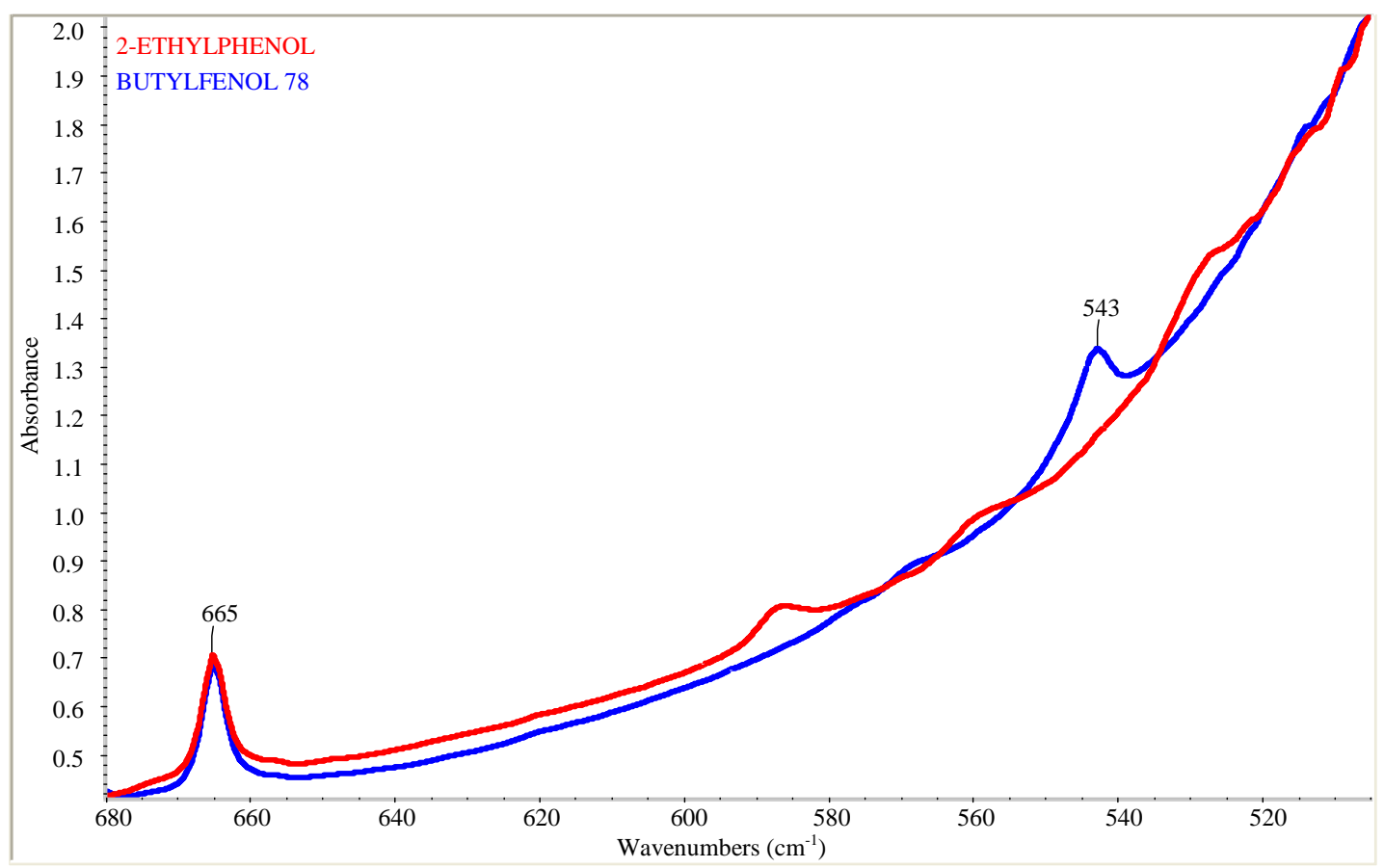

Figure 11. The FTIR spectra of 2-ethyl phenol (red line) and 2-n-butyl phenol (blue line) in the spectral region of $680-505 \mathrm{~cm}^{-1}$ (a thin film between $\mathrm{NaCl}$ windows).

\section{Acknowledgements}

This work was carried out thanks to the support of the long-term project for the conceptual development of the research organization No. 67985891.

\section{References}

[1] Buryan, P. and Macák, J. (1982) Partial Explanation of the Anomaly in the Relationship between the Logarithm of Retention and the Carbon Number of Monohydric Phenols. Journal of Chromatography A, 237, 381-388. http://dx.doi.org/10.1016/S0021-9673(00)97624-6

[2] Macák, J., Nabivach, V., Buryan, P. and Šindler, S. (1982) Dependence of Retentions Indices of Alkylbenzenes on Their Molecular Structure. Journal of Chromatography A, 234, 285-302. http://dx.doi.org/10.1016/S0021-9673(00)81867-1

[3] Straka, P. and Buryan, P. (2011) A Study of the Behavior of Alkyl Side Chains Phenols and Arenes in Polar and Nonpolar GC Stationary Phases. American Journal of Analytical Chemistry, 2, 324-331. http://dx.doi.org/10.4236/ajac.2011.23040

[4] Risby, T.H., Hsu, T.-B., Sehnert, S.S. and Bhan, P. (1990) Physicochemical Parameters of Individual Hexachlorobiphenyl Congeners. Environmental Science \& Technology, 24, 1680-1687. http://dx.doi.org/10.1021/es00081a009

[5] Janssen, F. (1982) Glass Capillary Gas Chromatography with Liquid Crystals. Chromatographia, 15, 33-37. http://dx.doi.org/10.1007/BF02269037

[6] García-Raso, A., Ballester, P., Bergueiro, R., Martínez, I., Sanz, J. and Jimeno, M.L. (1987) Estimation of the Polarity of Stationary Phases by Proton Nuclear Magnetic Resonance Spectroscopy Application to Phenyl and Methyl Silicones (OV and SE Series). Journal of Chromatography A, 402, 323-327. http://dx.doi.org/10.1016/0021-9673(87)80031-6

[7] Mathews, J.P. and Chaffee, A.L. (2012) The Molecular Representations of Coal—A Review. Fuel, 96, 1-14. http://dx.doi.org/10.1016/j.fuel.2011.11.025

[8] Howard, A., McIver, J. and Collins, J. (1994) HyperChem Computational Chemistry. Hypercube Inc., Waterloo.

[9] Becker, U. and Allinger, N.L. (1982) Molecular Mechanics. American Chemical Society, Washington DC.

[10] Allinger, N.L. and Yuh, Y.H. (1982) Quantum Chemistry Program Exchange. Indiana University, Bloomington.

[11] Allinger, N.L., Yuh, Y.H. and Lii, J.H. (1989) Molecular Mechanics. The MM3 Force Field for Hydrocarbons. Journal of the American Chemical Society, 111, 8551-8566. http://dx.doi.org/10.1021/ja00205a001 
Scientific Research Publishing (SCIRP) is one of the largest Open Access journal publishers. It is currently publishing more than 200 open access, online, peer-reviewed journals covering a wide range of academic disciplines. SCIRP serves the worldwide academic communities and contributes to the progress and application of science with its publication.

Other selected journals from SCIRP are listed as below. Submit your manuscript to us via either submit@scirp.org or Online Submission Portal.
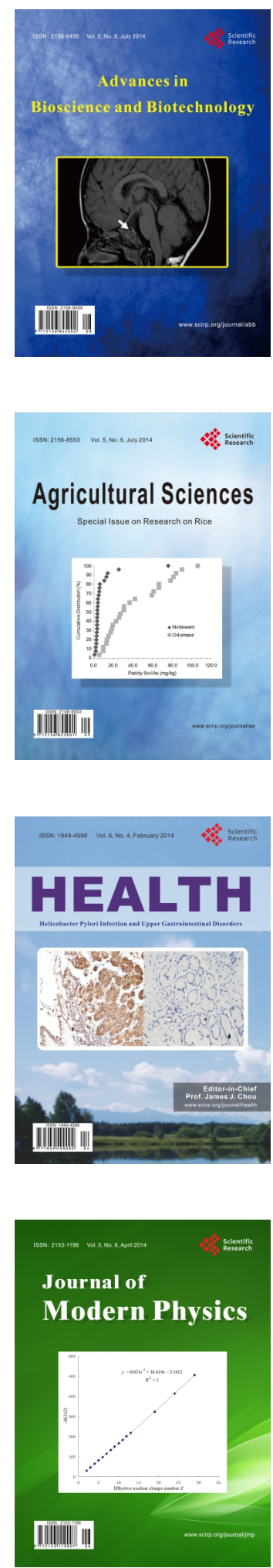
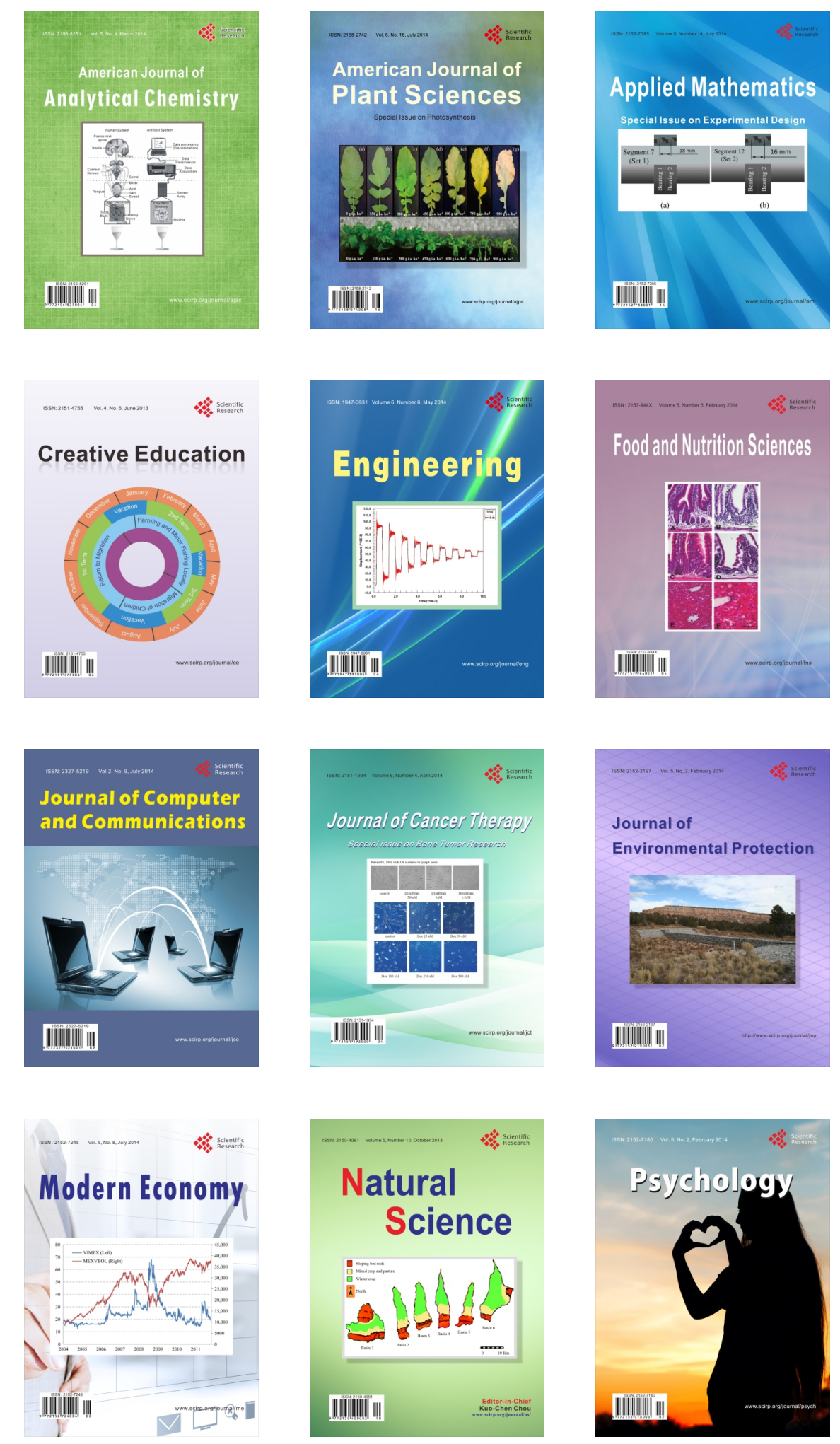